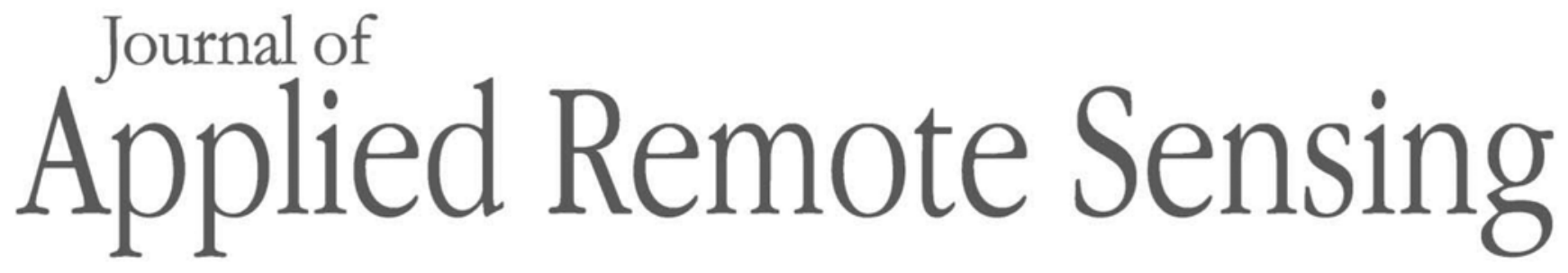

RemoteSensing.SPIEDigitalLibrary.org

\title{
Evaluation of VIIRS dust detection algorithms over land
}

\author{
Hai Zhang \\ Pubu Ciren \\ Shobha Kondragunta \\ Istvan Laszlo
}




\title{
Evaluation of VIIRS dust detection algorithms over land
}

\author{
Hai Zhang, ${ }^{\text {a,* Pubu Ciren, }}{ }^{\mathrm{a}}$ Shobha Kondragunta, ${ }^{\mathrm{b}}$ and Istvan Laszlo ${ }^{\mathrm{b}, \mathrm{c}}$ \\ a.M. Systems Group, College Park, Maryland, United States \\ ${ }^{b}$ National Oceanic and Atmospheric Administration/National Environmental Satellite, \\ Data, and Information Service, College Park, Maryland, United States \\ ${ }^{c}$ University of Maryland College Park, College Park, Maryland, United States
}

\begin{abstract}
This paper compares three dust detection algorithms over land that were developed for operational, near-real-time processing using the Suomi National Polar Orbiting Partnership Visible Infrared Imaging Radiometer Suite instrument. The three algorithm approaches use different spectral bands, namely deep blue bands, infrared (IR)-visible bands, and IR bands, and are applied for dust observed over dark as well as bright surfaces. The evaluations are performed both using case studies and AERONET matchup data over western CONUS-Mexico region and North Africa-Arabian Peninsula region. The deep blue-based algorithm is found to have the most false detections and its detection performance depends on the Sun-satellite geometries. Simulation analysis shows that there are three causes of this problem: surface reflectance, air mass factors, and phase functions in different geometries. The algorithm based on IR-visible bands has much less false detection than the deep blue bands-based algorithm and has better true positive detection than the IR-based algorithm. The IR bands-based algorithm performs well in the case studies over CONUS-Mexico region, but it fails to detect most of the dust cases over North Africa-Arabian Peninsula region. The results suggest that the IR-visible algorithm is the most suitable for the dust detection of the three algorithms with a small modification. Because the IR-visible algorithm is not able to detect all the dust pixels, detections from the deep blue algorithm only and those from the IR-visible algorithm with relaxed criteria are also provided but are distinguished with a lower quality. (1) The Authors. Published by SPIE under a Creative Commons Attribution 3.0 Unported License. Distribution or reproduction of this work in whole or in part requires full attribution of the original publication, including its DOI. [DOI: 10.1117/1.JRS.12.042609]
\end{abstract}

Keywords: dust; aerosol; Suomi National Polar Orbiting Partnership; Visible Infrared Imaging Radiometer Suite; deep blue; infrared; visible.

Paper 180474SS received May 31, 2018; accepted for publication Sep. 11, 2018; published online Oct. 8, 2018.

\section{Introduction}

Many satellite dust detection algorithms have been developed based on the absorption and scattering properties of dust and the underlying surfaces in different spectral bands. These algorithms are based on satellite measurements from different spectral bands, e.g., UV bands, ${ }^{1,2}$ deep blue bands, ${ }^{3}$ visible bands, ${ }^{4,5}$ and infrared (IR) bands. ${ }^{6-8}$

The Visible Infrared Imaging Radiometer Suite (VIIRS) sensor onboard Suomi National Polar Orbiting Partnership contains 22 different spectral bands ranging from 0.412 to $12.01 \mu \mathrm{m} .{ }^{9}$ The aerosol detection product (ADP) is one of the level 2 products derived from these bands. The algorithm uses the daytime VIIRS sensor measurements to detect smoke and dust over cloud/snow/ice free areas. ${ }^{3,10}$ The ADP algorithm detects smoke and dust at pixel level ( $750 \mathrm{~m}$ to $1.2 \mathrm{~km}$ depending on scan angle). It contains a deep blue algorithm and an IR-visible algorithm, which uses different bands for the detection. In the deep blue algorithm, dust pixels are identified through the use of thresholds tests of two indices, i.e., absorbing aerosol index (AAI) and dust smoke discrimination index (DSDI), which are derived from two deep blue bands (AAI) and a deep blue band and a short-wave IR band (DSDI). In the IR-visible algorithm, dust pixels are identified using the brightness temperature differences of three IR

*Address all correspondence to: Hai Zhang, E-mail: hai.zhang@noaa.gov 
bands and reflectances and indices from several visible bands. Details of the algorithms are described in Sec. 2. The algorithm generates smoke and dust mask from these two algorithms, i.e., if either of the algorithms determines a pixel is smoke or dust, the pixel is determined as smoke or dust. However, the performance of the deep blue-based algorithm is found to be poor over bright land surfaces for dust detection, as will be shown in the following sections. Therefore, to improve the performance of the dust detection product, an evaluation and comparison of the two VIIRS algorithms are performed over bright land surfaces, where the deep blue algorithm tends to have a lot of false dust detections. A third, IR-only dust detection algorithm is also introduced and evaluated, which is derived from the dust RGB method used on Meteosat Second Generation (MSG; dust and smoke detection with MSG SEVIRI RGB products). ${ }^{11}$

The three dust algorithms are described in Sec. 2 with an analysis of the ADP deep blue dust detection algorithm issues in Sec. 3. The dust detection using the IR-visible algorithm and IR algorithm for the dust cases introduced in Sec. 2 are presented in Sec. 4. Additional case studies from dust storms in North Africa and Arabian Peninsula are also discussed in Sec. 4. The validation of dust detection from the three different algorithms compared to AERONET matchup dataset is discussed in Sec. 5. The algorithms/products improvements are discussed in Sec. 6.

\section{Dust Detection Algorithms}

\subsection{VIIRS ADP Deep Blue Algorithm}

The ADP deep blue algorithm uses two indices to identify dust and smoke ${ }^{10}$ (NOAA/NESDIS/ STAR, 2016), which are AAI and DSDI, derived using the reflectance ratios from three bands: $0.41,0.44$, and $2.2 \mu \mathrm{m}$. AAI is defined as

$$
\mathrm{AAI}=-100\left[\log _{10}\left(\frac{R_{0.41}}{R_{0.44}}\right)-\log _{10}\left(\frac{R_{0.41}^{\prime}}{R_{0.44}^{\prime}}\right)\right],
$$

where $R_{0.41}$ and $R_{0.44}$ are the reflectances at the top of atmosphere (TOA) for the two deep blue bands, i.e., 0.41 and $0.44 \mu \mathrm{m}$, respectively, and $R^{\prime}$ s are the pure Rayleigh reflectances at the two bands. AAI generally increases with the increase of the absorbing aerosol loadings. The existence of dust or smoke can reduce the contrast between the two bands and, therefore, increase the value of AAI.

DSDI is defined as

$$
\mathrm{DSDI}=-10 \log _{10}\left(\frac{R_{0.41}}{R_{2.2}}\right),
$$

where $R_{0.41}$ and $R_{2.2}$ are the TOA reflectances of the two bands: 0.41 and $2.2 \mu \mathrm{m}$, respectively. As indicated by its name, this index is used to discriminate dust and smoke. The difference of DSDI between dust and smoke is that the $2.2-\mu \mathrm{m}$ band is sensitive to the existence of dust but not



Fig. $1 \mathrm{AAI}$ and DSDI features for different types of aerosols and clear sky. 
to the existence of smoke. Therefore, DSDI is larger for dust than that for smoke. The range of DSDI is between -18 and $3 .^{3}$

Figure 1 shows AAI versus DSDI for known samples of smoke, smog, dust, and clear sky pixels. AAI clearly separates absorbing aerosols from clear sky (AAI $<10$ being clear sky) and once absorbing aerosols are identified, DSDI can be used to separate smoke and smog from dust (DSDI $>-1$ being dust). While the DSDI shows overlap between smog and smoke, the composition of the aerosols tends to be regionally specific, which can be used to stratify the two aerosol types.

The detection of smoke and dust pixels in the ADP is based on the threshold tests given in the first part of Table 1 with the flowchart in Fig. 2(a). For example, if AAI is $>10.0$ and DSDI is $\geq 0.0$, a land pixel is determined as dust. The thresholds are obtained through the VIIRS matchup dataset with the Cloud-Aerosol Lidar and Infrared Pathfinder Satellite Observation (CALIPSO) measurements.

\subsection{VIIRS ADP IR-Visible Algorithm}

The VIIRS ADP algorithm also contains an algorithm that uses IR and visible bands for dust detection. ${ }^{10}$ The three IR bands used are 3.7, 10.8, and $12.0 \mu \mathrm{m}$. The main idea is to use the difference between the brightness temperatures (BT) of the three bands at TOA. When dust is present in a pixel, the BT differences of the bands are different from those of clear sky. In addition to the three IR bands mentioned above, the algorithm also uses several NIR-visible channels and derived indices to assist the dust detection:

$$
\begin{gathered}
\text { MNDVI }=\frac{\mathrm{NDVI}^{2}}{R_{0.67}^{2}}, \\
\mathrm{NDVI}=\frac{R_{0.86}-R_{0.67}}{R_{0.86}+R_{0.67}}, \\
\mathrm{RAT} 2=\frac{\mathrm{RAT}^{2}}{R_{0.48}^{2}},
\end{gathered}
$$

\begin{tabular}{|c|c|c|c|c|}
\hline Algorithm & Aerosol type & Threshold 1 & Threshold 2 & Others \\
\hline \multirow[t]{3}{*}{ Deep blue } & Dust & $\mathrm{AAI}>10.0$ & $\mathrm{DSDI} \geq 0.0$ & - \\
\hline & Thin smoke & $A A I \geq 5.0$ & $\mathrm{DSDI} \leq-3.0$ & - \\
\hline & Thick smoke & $A A I \geq 9.0$ & $\mathrm{DSDI} \leq-2.0$ & $0.2<R_{0.41}<0.4$ \\
\hline \multirow[t]{3}{*}{ IR-visible } & Thin dust & $\mathrm{BT}_{10.8}-\mathrm{BT}_{12.0} \leq-0.2$ & $\mathrm{BT}_{3.7}-\mathrm{BT}_{10.8} \geq 15$ & $\begin{array}{l}R_{1.38}<0.035 \\
\text { MNDVI }<0.8 \\
\text { RAT2 }>0.005\end{array}$ \\
\hline & Thin dust & - & $\mathrm{BT}_{3.7}-\mathrm{BT}_{10.8} \geq 20$ & - \\
\hline & Thick dust & $\mathrm{BT}_{10.8}-\mathrm{BT}_{12.0} \leq-0.2$ & $\mathrm{BT}_{3.7}-\mathrm{BT}_{10.8} \geq 20$ & $\begin{array}{l}R_{1.38}<0.035 \\
\mathrm{MNDVI}<0.2\end{array}$ \\
\hline \multirow[t]{2}{*}{ Dust RGB } & Dust & $\mathrm{BT}_{12.0}-\mathrm{BT}_{10.8}>0$ & $\begin{array}{c}\mathrm{BT}_{10.8}-\mathrm{BT}_{8.7}<0.5 \\
\text { in western CONUS-Mexico }\end{array}$ & $\mathrm{BT}_{10.8}>273$ \\
\hline & & & $\begin{array}{c}\mathrm{BT}_{10.8}-\mathrm{BT}_{8.7}<4 \\
\text { in North Africa and } \\
\text { Arabian Peninsula }\end{array}$ & \\
\hline
\end{tabular}

Table 1 Thresholds used to detect dust over land for the three algorithms. 
Zhang et al.: Evaluation of VIIRS dust detection algorithms over land

(a)

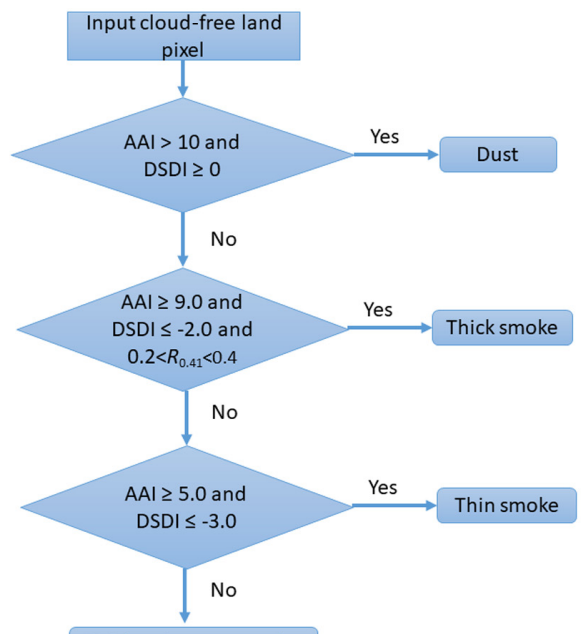

Neither dust nor smoke

(b)


Fig. 2 Flowcharts of the three algorithms: (a) deep blue, (b) IR-visible, and (c) dust RGB. 


$$
\mathrm{RAT} 1=\frac{R_{0.67}-R_{0.48}}{R_{0.67}+R_{0.48}}
$$

The second section of Table 1 and Fig. 2(b) show the criteria for the dust detection over land, where those for thin dust and thick dust are presented. There are two criteria for thin dust detection. If either one of them is satisfied, the corresponding pixel is set as thin dust.

The existence of dust perturbs the BT differences between different IR bands, i.e., the BT differences at TOA are different between dust pixels and clear sky pixels. The absorption of dust and the emissivity of surfaces are different in different IR bands. Therefore, the TOA BT differences demonstrate special features when the dust exists in the atmosphere. Here, in the IR-visible algorithm, three IR bands are selected, i.e., 3.7, 10.8, and $12.0 \mu \mathrm{m}$. The use of the visible bands can further differentiate dust from clouds. The purpose of the use of the squares in the MNDVI and RAT2 values is to make the quantities positive, as the signs of the values are found to be not important. The thresholds are determined through case studies of dust storms using VIIRS data.

\subsection{Thermal IR Algorithm from Dust RGB}

The third algorithm investigated is derived from the dust RGB method as used in MSG. ${ }^{11}$ In this algorithm, three IR bands are used: $8.5,10.8$, and $12.0 \mu \mathrm{m}$. The dust detection using these three bands was first introduced by Ackerman. ${ }^{6}$ To generate a dust RGB image, the red, green, and blue components of the image are set to be proportional to $\mathrm{BT}_{12.0}-\mathrm{BT}_{10.8}, \mathrm{BT}_{10.8}-\mathrm{BT}_{8.5}$, and $\mathrm{BT}_{10.8}$, respectively. The color of dust is magenta or pink in the derived dust RGB image. The reasons are as follows:

1. Surface emissivity in $10.8 \mu \mathrm{m}$ is similar to that in $12.0 \mu \mathrm{m}$, but dust absorption is higher in $10.8 \mu \mathrm{m}$. Therefore, BT at TOA in $12.0 \mu \mathrm{m}$ is higher than that in $10.8 \mu \mathrm{m}$, i.e., large red component in dust RGB image.

2. Surface emissivity in $10.8 \mu \mathrm{m}$ is higher than that in $8.5 \mu \mathrm{m}$, but dust absorption is also higher in $10.8 \mu \mathrm{m}$. Therefore, BT at TOA in $8.5 \mu \mathrm{m}$ is close to that in $10.8 \mu \mathrm{m}$, i.e., small green component in dust RGB image.

3. Surface is usually hot in daytime over desert surface, i.e., large blue component.

Due to these three factors, a dust region has large red, small green, and large blue, which looks as magenta or pink color.

Based on these ideas, a dust detection algorithm using thresholds is designed. The third section of Table 1 and Fig. 2(c) show the thresholds used in this work, which are determined by visual inspection of images of BT and BT differences from several dust cases over the regions of interest.

\section{Issues in VIIRS ADP Deep Blue Algorithm}

Although the VIIRS ADP deep blue algorithm performs well over ocean, problems are found for the detections over land, especially over bright surfaces. The problems appear as false detections of clear sky as dust, undetected dust storms, and geometry dependence of the detections. They are demonstrated in three case studies of dust storms in the western CONUS, which are located close to the borders of New Mexico, Texas, and Mexico. Two cases are the same dust storm observed in different time on December 17, 2016, i.e., 19:02 UTC and 20:43 UTC. The third case is on March 31, 2017, 19:52 UTC. The RGB true color images for the three cases are shown in Figs. 3(a)-3(c), which are generated using the Rayleigh corrected TOA reflectance of the three VIIRS visible bands: red $(0.67 \mu \mathrm{m})$, green $(0.55 \mu \mathrm{m})$, and blue $(0.48 \mu \mathrm{m})$. The plumes of dust storm can be seen in the true color RGB images.

Figures 3(d)-3(f) show the dust mask (in yellow-brown) derived from the VIIRS ADP deep blue algorithm overlaid on top of the true color images. The color of the dust mask is proportional to the values of AAI, i.e., the darker colors have higher AAI values. In the first image, only a very small portion of the dust storm is identified, while most part of the dust storm is not 



Fig. 3 True color RGB images of three dust storm observations from VIIRS: (a) December 17, 2016, 19:02 UTC, (b) December 17, 2016, 20:43 UTC, and (c) March 31, 2017, 19:52 UTC, and the corresponding dust mask (in yellow-brown) using the deep blue algorithm. The locations of AERONET sites Sevilleta and Tucson are plotted in the figures, which are the only sites report the measurements at the satellite overpass times. Both report low AOD values: AOD at Sevilleta in (a) and (d) is 0.02 and AOD at Tucson in (c) and (f) is 0.19.

detected. In the second image, no pixel in the dust storm region is detected. But the area in the west to the south of Arizona and California border is detected as dust incorrectly, even though the area looks clear in the corresponding true color RGB image [Fig. 3(b)]. In the third image, large clear areas are detected as dust incorrectly, while the dust storm area is also detected correctly.

AERONET aerosol optical depth (AOD) was examined to further confirm the visual inspections. However, only Sevilleta in Fig. 3(a) and Tucson in Fig. 3(c) have AOD measurements at the time of the satellite overpasses. Both sites observed low AOD: Sevilleta has AOD 0.02 and Tucson has AOD 0.19. None of them is in the dust storm regions or false detection regions. Therefore, the AERONET measurements do not help much in the analysis of these case studies.

Although the three VIIRS observations are over the same area, the observation geometries are different. Figure 4 shows the overpass tracks of the SNPP satellite which carries the VIIRS sensor. The first overpass of SNPP on December 16, 2016, is to the east of the area of interest, i.e., New Mexico/Texas/Mexico border, while the Sun shines from the west. Therefore, the geometry of the Sun-satellite is in forward reflection geometry, which is defined as relative azimuth angle $>90 \mathrm{deg}$. The second overpass for the same day is to the west of the area of interest and the corresponding geometry is in backward reflection geometry. For the third case, i.e., on March 31, 2017, the area of interest is close to the nadir of the satellite overpass. In the following discussion, we refer the three cases as caseF, caseB, and caseN so that their geometrical characters are identified. Figure 5 shows an illustration of the three geometries.

The differences of the geometries in the three cases along with the underlying surface reflectance are the causes of the differences in the dust detection. A simulation study is performed to demonstrate the causes of the detection problems. As shown in Fig. 6, the pink box regions are selected for the simulation, which have the same latitude-longitude boundaries in the three images. The parameters for the three regions are shown in Table 2. The solar zenith angles $\left(\theta_{s}\right)$, satellite view zenith angles $\left(\theta_{v}\right)$, and relative azimuth angles $(\varphi)$ for the three cases are obtained through averaging the corresponding parameters for each pixel within the boxes. 




Fig. 4 SNPP tracks for two overpasses on December 16, 2016, and for one overpass on March 31, 2017.
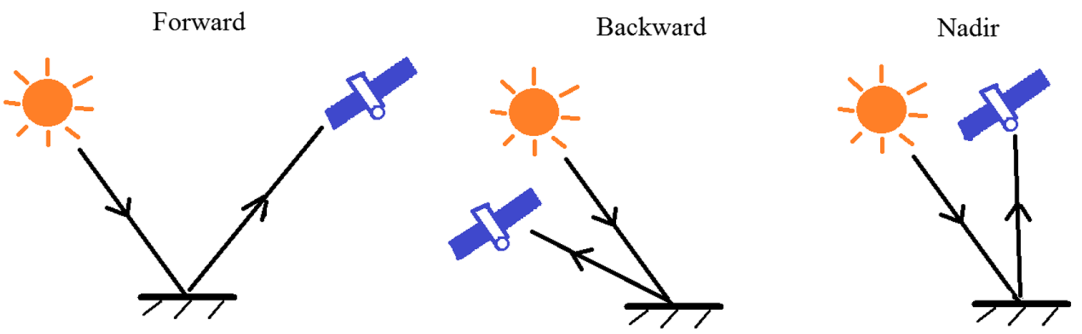

Fig. 5 An illustration of the geometries for the three cases, i.e., caseF, caseB, and caseN.

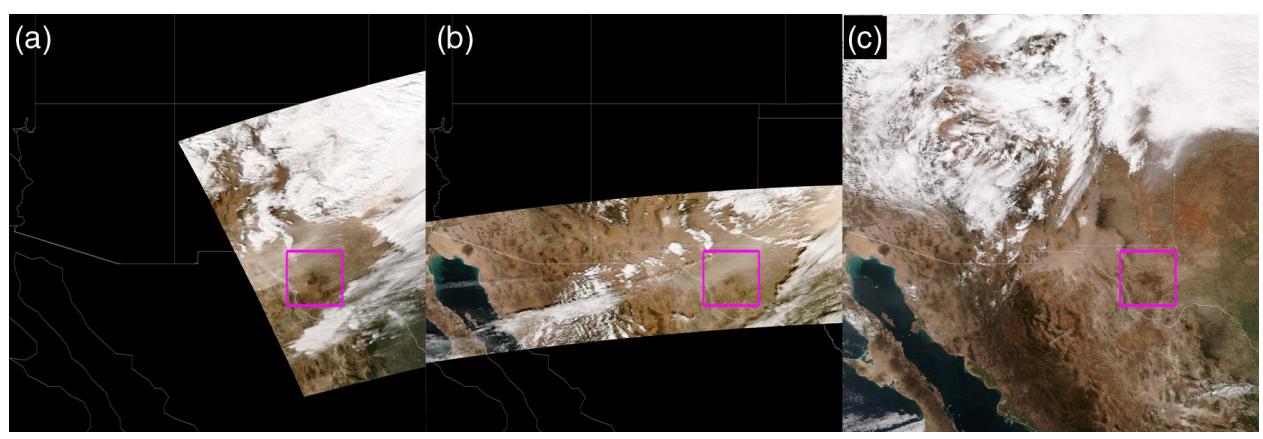

Fig. 6 Regions for the simulation study are shown in the pink boxes, which have the same latitude-longitude boundaries in the three cases: (a) CaseF, (b) CaseB, and (c) CaseN.

Table 2 Parameters used in the simulation study.

\begin{tabular}{lcccc}
\hline \hline Case & Overpass time & $\begin{array}{c}\text { Latitude longitude } \\
\text { bound }\end{array}$ & Geometries $\left(\theta_{s}, \theta_{v}, \varphi\right)$ & $\begin{array}{c}\text { Surface reflectance } \\
\text { at } 0.41,0.44, \\
\text { and } 2.12 \mu \mathrm{m}\end{array}$ \\
\hline caseF & 201612171902 UTC & $298^{\circ} \mathrm{N}$ to $31.8^{\circ} \mathrm{N}$ & $\begin{array}{c}\text { Forward }(54.27 \mathrm{deg}, \\
65.26 \mathrm{deg}, 110.86 \mathrm{deg}\end{array}$ & $0.050,0.056,0.20$ \\
& & $105^{\circ} \mathrm{W}$ to $103^{\circ} \mathrm{W}$ & & \\
caseB & 201612172043 UTC & & $\begin{array}{c}\text { Backward }(60.37 \mathrm{deg}, \\
57.52 \text { deg, } 55.80 \mathrm{deg})\end{array}$ & $0.070,0.081,0.28$ \\
& & & $\begin{array}{c}\text { Close to nadir }(29.11 \mathrm{deg}, \\
13.29 \text { deg, 128.71 deg) }\end{array}$ & $0.076,0.087,0.27$ \\
\hline \hline
\end{tabular}


The surface reflectances at 0.41 and $0.44 \mu \mathrm{m}$ are obtained from a surface reflectance database derived from multiyear VIIRS observations using a method similar to that in the MODIS deep blue algorithm ${ }^{12}$ and VIIRS bright surface algorithm. ${ }^{13}$ In this database, the surface reflectance is linearly dependent on the scattering angle with different coefficients for forward and backward reflection geometries. Because of the geometry dependence, the surface reflectance close to the nadir is the largest of the three cases, while that in the forward reflection direction is the smallest.

Two aerosol models are used in the simulation: a dust model and a smoke model, whose optical properties are the same as those used by Levy et al. ${ }^{14}$ and Laszlo and Liu. ${ }^{15}$ The radiative transfer model used for the simulation is $6 \mathrm{SV}$ model. ${ }^{16,17}$ Figure 7 shows the simulation results, which are the plots of the AAI variation with respect to the variation of AOD for both dust and smoke models in the three different geometries. The figures show that the dynamic ranges of AAI are different for the three cases for the same variations of the AOD change from 0 to 2.0. The forward reflection direction has the largest variation of AAI and, therefore, best suited for dust detection. The variation of AAI in the area close to the nadir is very small, and therefore, the dust detection in this geometry has the worst performance. This can explain the false detection in the caseN shown in Fig. 3(f): in the geometry close to nadir, AAI is not sensitive to AOD change, AAI is high even if there is no aerosol loading, and therefore, it is hard to tell the difference between clear sky area and dust area by looking at the AAI field. Even though AAI has some variation in the backward reflection geometry as shown in Fig. 7, the variation is much smaller than that in the forward reflection geometry. In the ADP algorithm, a threshold of AAI $=10$ is used to separate dust pixels and clear sky pixels. In the backward reflection geometry, AAI values are much lower than the threshold. Therefore, the dust plume is not detected in the second case in Fig. 3.

In all the three geometries, only thick dust with AOD close to 2.0 in caseF can have AAI close to the threshold 10. One possible reason is that the AAI threshold is determined through actual observed data using CALIPSO matchups. ${ }^{3}$ The aerosol models used in the simulation may be different from the actual aerosol models and hence the simulated values can be different. In addition, the surface reflectances used in the simulation are the averages of all the pixels within the boxes, while individual VIIRS pixels can be higher or lower than the averages and, therefore, can have different AAI values.
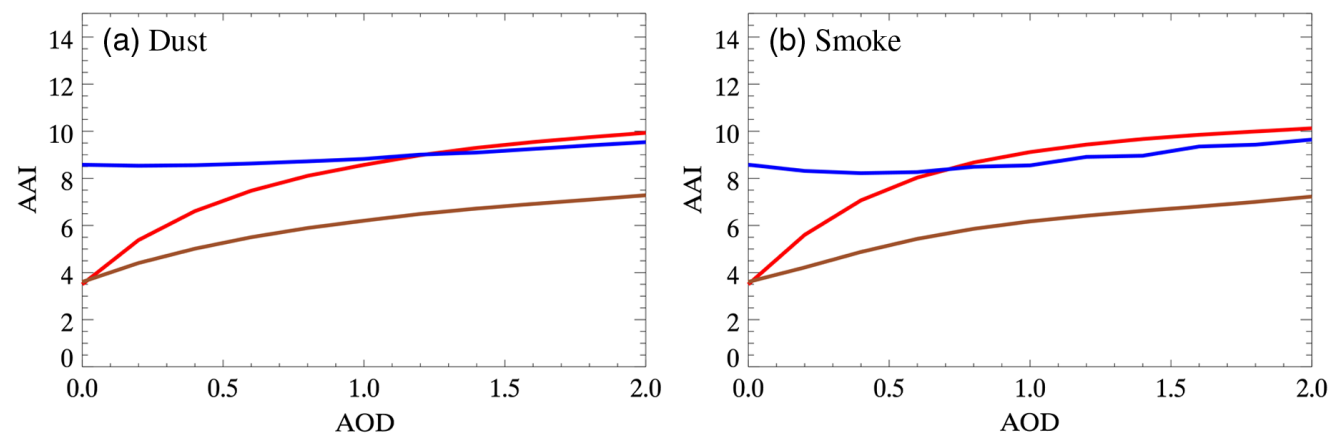

Fig. 7 AAl variation versus AOD variation for (a) dust and (b) smoke aerosol models for the three cases: red: caseF, brown: caseB, and blue: caseN.

Table 3 Surface reflectances, air mass factors, and phase functions for the three cases.

\begin{tabular}{lccc}
\hline \hline Case & $\begin{array}{c}\text { Surface reflectance } \\
(0.41 \text { and } 0.44 \mu \mathrm{m})\end{array}$ & $\begin{array}{c}\text { Air mass factor } \\
\left(1 / \cos \theta_{s}+1 / \cos \theta_{v}\right)\end{array}$ & Phase function \\
\hline caseF & 0.050 .056 & 4.06 & $\sim 0.3$ (scattering angle $88 \mathrm{deg}$ ) \\
caseB & $0.070,0.081$ & 3.83 & $<0.2$ (scattering angle 132 deg) \\
caseN & $0.076,0.087$ & 2.17 & $<0.2$ (scattering angle 141 deg) \\
\hline \hline
\end{tabular}


Since the TOA reflectance is related to the surface reflectance contribution and atmosphere contribution, the difference in the three cases must originate from the difference in the surface and atmosphere for the three cases. Table 3 shows three reasons contributing to the differences in the AAI change versus AOD change in Fig. 7, i.e., surface reflectance, air mass factor, and phase functions. In addition to the surface reflectance, which has been mentioned before, the air mass factor is the smallest for the caseN, which is only about a half of the other two. The phase function of the aerosols in the caseN is also smaller than that of caseF. These three reasons make the caseN's TOA reflectance of the two deep blue bands the least sensitive to the aerosol loadings, and therefore, its AAI is also the least sensitive. Comparing the other two cases, caseB has higher surface reflectance, less air mass factor, and smaller phase function, and therefore, the corresponding AAI is not as sensitive to AOD as that of caseF.

The above case studies and analysis indicate that AAI sensitivity of the aerosol loadings is a function of the surface reflectance and Sun-satellite geometry. Therefore, it is not appropriate to use a universal AAI threshold to discriminate dust pixels from clear sky pixels. In the areas close to the nadir with high surface reflectance, AAI is not sensitive enough such that there is not much difference between dust and clear sky pixels. Therefore, AAI is not suitable to be used for the dust detection in such geometries and surface reflectance combinations.

\section{Detect Dust Using IR Bands}

\subsection{Western CONUS and Mexico Region}

In this section, an investigation of the dust detection using the other two algorithms for the same three cases in Sec. 3 is performed using IR or IR-visible bands. Figure 8 shows the dust RGB images for the three cases in Sec. 3, generated using the dust RGB technique as described in Sec. 2. The dust plumes in the three cases are all shown as magenta/pink color and can be identified visually, although some land surfaces are also shown as pink color, indicating that the variations of land surface temperature and emissivity interfere with the dust signals. In dust RGB images, different color represents different features of the atmosphere and surface; in addition to the magenta/pink for dust, dark brown represents high clouds, brown represents low mid-level clouds, blue represents thin clouds, and white/cyan/purple/pink represents surface with different temperatures. When surfaces are hot, the color is closer to white/cyan, while it is closer to pink when surfaces are cold. Therefore, the dust plume is more prominent over hot surfaces, e.g., over tropical desert.

Dust mask can be derived through applying the thresholds described in Sec. 2 (Table 1). The results are shown in Fig. 9. The dust masks using this algorithm can identify most of the dust plumes in all the three cases. In addition, there is almost no false detection of clear sky as dust. There is also no significant geometry dependence, as the algorithm works equally well for all the three cases, even though the geometries are different significantly between them.

To demonstrate the ADP IR-visible algorithm, Fig. 10 shows the dust masks for the three cases using this algorithm, which look similar to those using the IR algorihm for dust RGB. However, some false detections can be seen in the second and the third cases using this method,



Fig. 8 Dust RGB images for the three cases in Sec. 3: (a) CaseF, (b) CaseB, and (c) CaseN. 


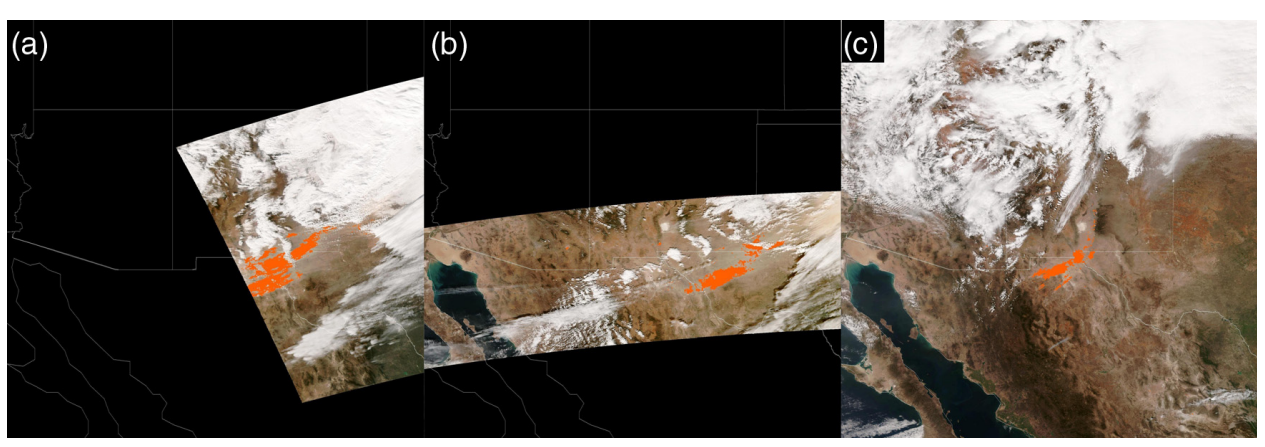

Fig. 9 Dust mask (in brown) derived using the dust RGB thresholds in Table 1 for the three cases in western CONUS: (a) CaseF, (b) CaseB, and (c) CaseN.

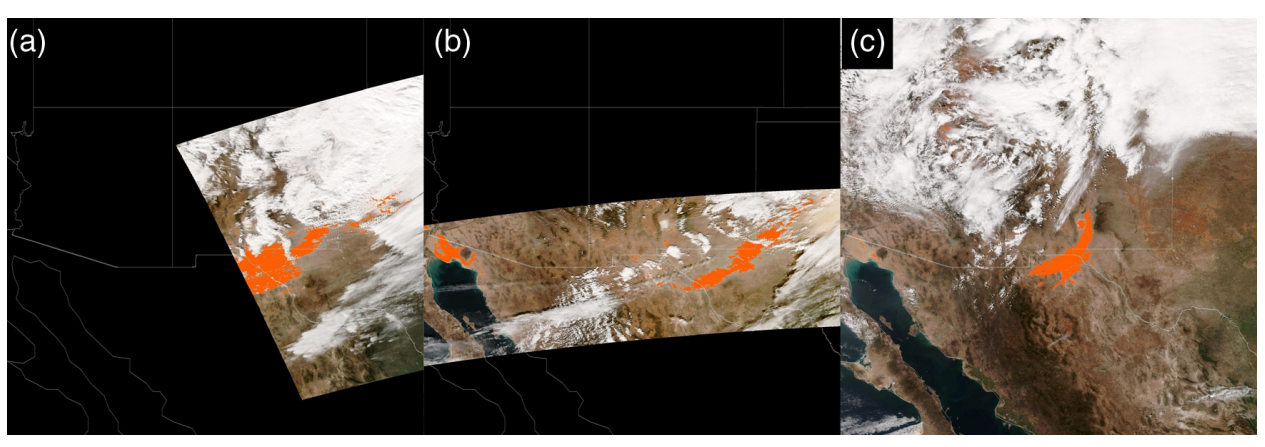

Fig. 10 Dust mask generated using the IR-visible algorithm: (a) CaseF, (b) CaseB, and (c) CaseN.

where very high surface reflectances are observed as well as some missed detections. The plume is clearly more widespread than the detected one in both case 1 and case 2 .

\subsection{North Africa and Arabian Peninsula Region}

North Africa and Arabian Peninsula region is an area where dust storms occur frequently. ${ }^{18,19}$ This section shows two case studies using the three algorithms in this region.

For the IR algorithm from dust RGB, it is found that a slightly different threshold should be used in the North Africa and Arabian Peninsula region to have good detection performance. As shown in Table 1, the BT differences between 10.8 and 8.7 is set to be $<4$ in this region, instead of 0.5 in the western CONUS-Mexico region. This may be due to different surface types and/or dust types between these two regions, ${ }^{20,21}$ which can cause the BT difference between the two bands not to be the same in different regions. ${ }^{22}$

Figures 11 and 12 show images of two dust cases over North Africa and Arabian Peninsula region, where true color RGB images and the dust masks from the three algorithms are plotted. In both cases, dust mask from the deep blue algorithm tends to overestimates the dust areas, since some areas with no dust signature in the true color images are identified as dust in the ADP deep blue algorithm. The results from the two IR-based algorithms look quite similar. However, the IR-visible and IR algorithms seem to underestimate the area of dust region. For example, in Fig. 11, the areas in Niger look like dust in RGB, but they are not detected in the IR-visible and IR algorithms.

Table 4 shows the AERONET measurements of AOD and Angstrom exponent (AE). We consider dust exists when AOD $>0.3$ and $\mathrm{AE}<0.6 .{ }^{10}$ In the 20150909 case, all three algorithms detect dust over the sites SEDE_BOKER, Eilat, and Cairo and none detects over Kuwait University (as shown in Fig. 11). The detections are in agreement with AERONET measurements. In the case of 20130823, all three algorithms detect dust at Tamanrasset and do not detect dust at Oujda, which are in agreement with the AERONET measurements. At Zinder_Airport, deep blue algorithm detects dust but the other two algorithms do not detect. However, AE at this 


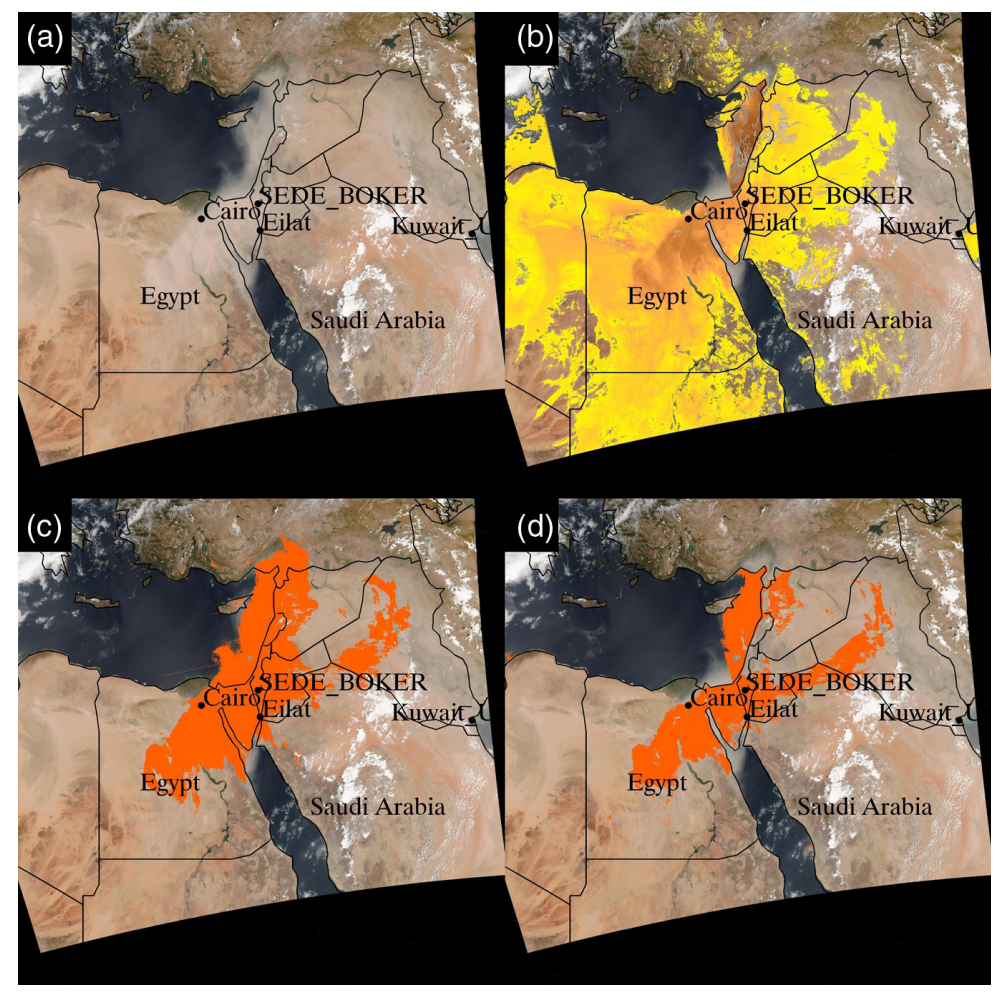

Fig. 11 Dust detection on September 9, 2015, case over North Africa and Arabian Peninsula. The images are (a) VIIRS true color RGB, (b) dust detection from ADP deep blue algorithm, (c) dust mask from IR algorithm, and (d) dust mask from IR-visible algorithm.

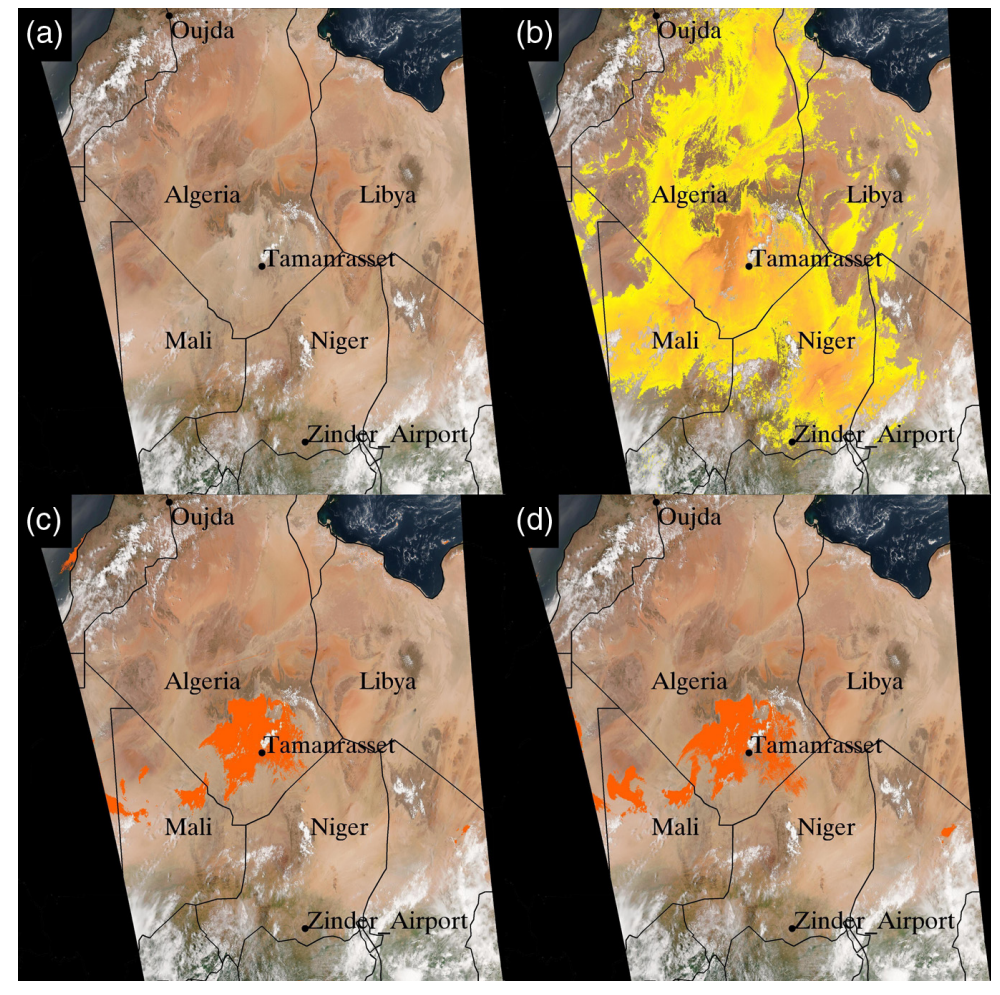

Fig. 12 Same as Fig. 11, but for the case in North Africa on August 23, 2013: (a) VIIRS true color RGB, (b) dust detection from ADP deep blue algorithm, (c) dust mask from IR algorithm, and (d) dust mask from IR-visible algorithm. 
Table 4 AERONET AOD and AE for the case studies over North Africa and Arabian Peninsula region. The locations of the AERONET sites are shown in Figs. 11 and 12.

\begin{tabular}{lccccc}
\hline \hline Case & Site name & Latitude & Longitude & AOD & AE \\
\hline 20150909 & SEDE_BOKER & 30.85 & 34.78 & 3.17 & -0.19 \\
& Kuwait University & 29.32 & 47.97 & 0.71 & 0.64 \\
& Eilat & 29.50 & 34.91 & 3.65 & 0.05 \\
\multirow{2}{*}{20130823} & Cairo & 30.08 & 31.29 & 4.51 & 0.05 \\
& Tamanrasset & 22.79 & 5.53 & 1.22 & 0.03 \\
& Zinder_Airport & 13.77 & 8.99 & 0.55 & 0.60 \\
& Oujda & 34.65 & -1.90 & 0.30 & 0.73 \\
\hline \hline
\end{tabular}

site is exactly at the value we set for dust determination (0.6). Therefore, it is hard to say which algorithm provides better detection at this site.

\section{Evaluation Using the AERONET Matchup Data}

In this section, the three algorithms are evaluated using the matchup dataset between VIIRS and AERONET $^{23}$ spectral AOD data for the year 2015. The matchup criteria are as follows: (1) VIIRS pixels are located within 27.5-km circle of the AERONET site and (2) the AERONET data are within $\pm 30 \mathrm{~min}$ of the satellite overpass. The AERONET level 1.5 data are used in this study. The three dust detection algorithms were applied to this dataset on cloud-free pixels for both western CONUS area and Northern Africa and Arabian Peninsula regions. The criteria for positive AERONET dust detection are: AOD $>0.3$ and $\mathrm{AE}<0.6$. Negative dust detection, i.e., non-dust aerosol or low aerosol loading, is determined AERONET (1) AOD $>0.3$ and $\mathrm{AE}>1.1$ or (2) AOD $\leq 0.3$. Otherwise, the aerosol type is undetermined. The criteria for a positive VIIRS detection are: (1) total number of matchup cloud-free VIIRS pixels is $>800$, which is about $20 \%$ of all the pixels within the matchup area and (2) more than half of the cloud-free pixels are detected as dust from the VIIRS dust detection algorithm. The dust detections from VIIRS are compared against AERONET detections and are classified into five categories: (1) true positive-both VIIRS and AERONET detections are positive, (2) false positive-VIIRS positive and AERONET negative, (3) false negative-VIIRS negative and AERONET positive, (4) true negative-both are negative, and (5) undetermined-AERONET detection is undetermined.

The statistics of the results using the AERONET matchup data is shown in Table 5. Over western CONUS-Mexico region, there are only nine points that are determined by the AERONET as dust and none of them are detected by any of the three algorithms. Visual

Table 5 Statistics of dust detection for the three algorithms on the AERONET matchup data in 2015

\begin{tabular}{|c|c|c|c|c|c|c|c|}
\hline & Algorithms & $\begin{array}{c}\text { True } \\
\text { positive }\end{array}$ & $\begin{array}{c}\text { True } \\
\text { negative }\end{array}$ & $\begin{array}{l}\text { False } \\
\text { positive }\end{array}$ & $\begin{array}{c}\text { False } \\
\text { negative }\end{array}$ & Undetermined & $\begin{array}{c}\text { Total } \\
\text { matchups }\end{array}$ \\
\hline \multirow{3}{*}{$\begin{array}{l}\text { Western } \\
\text { CONUS-Mexico }\end{array}$} & Deep blue & 0 & 4579 & 71 & 9 & 7 & \multirow[t]{3}{*}{4666} \\
\hline & IR-visible & 0 & 4616 & 34 & 9 & 7 & \\
\hline & IR & 0 & 4650 & 0 & 9 & 7 & \\
\hline \multirow{3}{*}{$\begin{array}{l}\text { North } \\
\text { Africa-Arabian } \\
\text { Peninsula }\end{array}$} & Deep blue & 338 & 3709 & 252 & 674 & 281 & \multirow[t]{3}{*}{5254} \\
\hline & IR-visible & 208 & 3890 & 71 & 804 & 281 & \\
\hline & IR & 35 & 3867 & 94 & 977 & 281 & \\
\hline
\end{tabular}


inspections of the nine cases over the satellite images show that none of them look like dust, which means that the AERONET test criteria may also have false detections. The low number of dust cases is probably due to that the dust storms over western CONUS and Mexico region usually last in short-time period and in a relative small region. ${ }^{24}$ Therefore, it is difficult for VIIRS and AERONET to capture dust storms simultaneously. On the other hand, there are more dust cases over North Africa and Arabian Peninsula region, i.e., 1012 in total matchups. The deep blue algorithm has the most number of true positive detections (338), which is followed by the IR-visible algorithm (208). Although IR algorithm seems to have good detection in the previous case studies, the number of true positive detection over North Africa and Arabian Peninsula region is very low compared to the other two algorithms. All three algorithms have large number of false negative detections.

In both regions, the deep blue algorithm has the highest number of false positive detections, which is in agreement with our previous case studies. The IR algorithm does not have false positive detection over western CONUS-Mexico region, but it has 94 false positive over North Africa-Arabian Peninsula region. The IR-visible algorithm has some false positive detections over both areas.

\section{Algorithm Modification}

The evaluations in Sec. 5 show that all three dust detection algorithms have their drawbacks. The deep blue algorithm tends to have large amount of false positive detections, while dust RGB algorithm detects the least true positive dust. Since dust RGB algorithm tends to have the least true positive detections over North Africa-Arabian Peninsula region, this algorithm is not suggested to be used, but the criteria in the algorithm can be used to improve the IR-visible algorithm.

One possible improvement to the algorithm is to improve the IR-visible algorithm by adding one more criteria from dust RGB algorithm over western CONUS-Mexico region: $\mathrm{BT}_{10.8}-\mathrm{BT}_{8.7}<0.5$. After adding this criterion, the IR-visible algorithm can remove some of the false positive detections over western CONUS-Mexico region. While not shown, we found that the number of false positive detections over this region reduces from 34 to 8 for the case study in Sec. 6.

Classification of pixels into different qualities can be used to represent different confidence in the detections. The following rules are suggested based on the previous results: (1) if a pixel is detected as dust from IR-visible algorithm, the detection quality is set as high. (2) If a pixel is not detected as dust from IR-visible algorithm but detected as dust from deep blue algorithm, the detection quality is set as low. (3) Since IR-visible algorithm has two set of rules using IR bands, i.e., first two columns in Table 2, and the criteria require both rules should be satisfied, a relaxed rule can require either of the columns is satisfied and set the quality as low if not both are satisfied. With the inclusion of low-quality pixels, the possibility of true positive detection increases.

\section{Conclusions}

A comparison of three dust detection algorithms is performed in this work through case studies and analysis. The VIIRS ADP algorithm using deep blue bands results in large areas of false detection over bright surfaces. Both the case studies and the analysis show that the deep blue algorithm does not work well in some geometries and surface reflectance combinations. The two IR-based algorithms have similar detection results in the case studies. However, the ADP IR-visible algorithm tends to have some false detection over very bright areas in the western CONUS region.

The ADP deep blue algorithm was designed following the Ozone Monitoring Instrument (OMI) aerosol index (AI) algorithm. ${ }^{25}$ The reasons that it does not work as well as OMI AI are following. OMI AI works in the UV bands, in which the surface reflectance is much lower than those in the deep blue bands. The aerosol absorption and Rayleigh reflectance are also much higher in UV bands than those in deep blue bands, which are critical in the mechanism of the AAI usage in dust detections. 
The comparison using the AERONET matchup dataset shows similar results for the deep blue algorithm. However, the IR algorithm has very low true positive detections over North Africa-Arabian region. The IR-visible algorithm seems to be the best in terms of true positive and false positive detections. Although the IR-visible algorithm does not have as many true positive as the deep blue algorithm over North Africa-Arabian region, it has much less false positive detection than that of the deep blue algorithm.

The results of this work demonstrate that the deep blue algorithm is not suitable for dust detection over bright land surfaces by itself and, therefore, should be designated low quality. Of the three algorithms, IR-visible algorithm with a modification performs the best and is suggested to be used for dust detection as high-quality detections. Other than those detected by the IR-visible algorithm, detections from the deep blue and the relaxed IR-visible algorithm are set as low quality.

The Advanced Baseline Imager onboard the geostationary satellite GOES-R series has similar bands as VIIRS, except that GOES-R does not have the deep blue channels. ${ }^{26}$ Therefore, the IR-visible algorithm will be applied to the GOES-R data. Investigations and examinations are being performed on the GOES-R data. A couple of differences between the two satellites may affect the direct application of the algorithm on GOES-R: (1) Sun-satellite geometry and (2) shifts in spectral response functions between the two satellite sensors. Therefore, the thresholds of the criteria need to be inspected and geometry dependence of the thresholds will also need to be investigated.

\section{Acknowledgments}

This work was supported by the NOAA JPSS program. The authors thank the AERONET principal investigators and site managers for providing the data used in this work. The authors thank the Rebekah Esmaili for the NOAA internal review of the paper. The contents in this paper are solely the opinions of the authors and do not constitute a statement of policy, decision, or position on behalf of NOAA or the U.S. Government.

\section{References}

1. N. C. Hsu et al., "Comparisons of the TOMS aerosol index with sun-photometer aerosol optical thickness: results and applications," J. Geophys. Res. 104(D6), 6269-6279 (1999).

2. O. Torres et al., "Aerosol properties from EP-TOMS near UV observations," Adv. Space Res. 29, 1771-1780 (2002).

3. P. Ciren and S. Kondragunta, "Dust aerosol index (DAI) algorithm for MODIS," J. Geophys. Res. Atmos. 119(8), 4770-4792 (2014).

4. S. D. Miller, "A consolidated technique for enhancing desert dust storms with MODIS," Geophys. Res. Lett. 30(20), 2071 (2003).

5. I. Jankowiak and D. Tanré, "Satellite climatology of Saharan dust outbreaks: method and preliminary results," J. Clim. 5, 646-656 (1992).

6. S. A. Ackerman, "Remote sensing aerosols using satellite infrared observations," J. Geophys. Res. 102(D14), 17069-17079 (1997).

7. A. Darmenov and I. N. Sokolik, "Identifying the regional thermal-IR radiative signature of mineral dust with MODIS," Geophys. Res. Lett. 32, L16803 (2005).

8. R. A. Hansell et al., "Simultaneous detection/separation of mineral dust and cirrus clouds using MODIS thermal infrared window data," Geophys. Res. Lett 34, L11808 (2007).

9. D. Hillger et al., "First-light imagery from Suomi NPP VIIRS," Bull. Am. Meteorol. Soc. 94, 1019-1029 (2013).

10. NOAA/NESDIS/STAR, "JPSS aerosol detection product algorithm theoretical basis document (ATBD)," 2016, https://www.star.nesdis.noaa.gov/jpss/documents/ATBD/ATBD_ EPS_Aerosol_ADP_v1.1.pdf (19 September 2018).

11. "Dust \& Smoke Detection with MSG SEVIRI RGB Products," https://www.eumetsat.int/ website/home/Data/Training/TrainingLibrary/DAT_2042630.html (19 September 2018).

12. N. C. Hsu et al., "Enhanced deep blue aerosol retrieval algorithm: the second generation," J. Geophys. Res. Atmos. 118, 9296-9315 (2013). 
13. H. Zhang et al., "An enhanced VIIRS aerosol optical thickness (AOT) retrieval algorithm over land using a global surface reflectance ratio database," J. Geophys. Res. 121, 10717 10738 (2016).

14. R. C. Levy, L. A. Remer, and O. Dubovik, "Global aerosol optical properties and application to Moderate Resolution Imaging Spectroradiometer aerosol retrieval over land," J. Geophys. Res. 112, D13210 (2007).

15. I. Laszlo and H. Liu, "NOAA NESDIS center for satellite applications and research EPS aerosol optical depth (AOD) algorithm theoretical basis document," v 3.0 (2016).

16. S. Y. Kotchenova et al., "Validation of a vector version of the $6 \mathrm{~S}$ radiative transfer code for atmospheric correction of satellite data. Part I: path radiance," Appl. Opt. 45(26), 6762-6774 (2006).

17. S. Y. Kotchenova and E. F. Vermote, "Validation of a vector version of the $6 \mathrm{~S}$ radiative transfer code for atmospheric correction of satellite data. Part II: homogeneous Lambertian and anisotropic surfaces," Appl. Opt. 46(20), 4455-4464. (2007).

18. J. Prakash et al., "The impact of dust storms on the Arabian Peninsula and the Red Sea," Atmos. Chem. Phys. 15, 199-222 (2015).

19. D. Kim et al., "Source, sinks, and transatlantic transport of North African dust aerosol: a multi-model analysis and comparison with remote-sensing data," J. Geophys. Res. 119, 6259-6277 (2014).

20. M. C. Reheis et al., "Compositions of modern dust and surface sediments in the Desert Southwest, United States," J. Geophys. Res. 114, F01028 (2009).

21. P. Formenti et al., "Regional variability of the composition of mineral dust from western Africa: results from the AMMA SOP0/DABEX and DODO field campaigns," J. Geophys. Res. 113, D00C13 (2008).

22. I. N. Sokolik, "The spectral radiative signature of wind-blown mineral dust: implications for remote sensing in the thermal IR region," Geophys. Res. Lett. 29(24), 7-1-7-4 (2002).

23. B. N. Holben et al., "AERONET - a federated instrument network and data archive for aerosol characterization," Remote Sens. Environ. 66(1), 1-16 (1998).

24. D. Q. Tong et al., "Long-term dust climatology in the western United States reconstructed from routine aerosol ground monitoring," Atmos. Chem. Phys. 12, 5189-5205 (2012).

25. O. Torres et al., "Aerosols and surface UV products from ozone monitoring instrument observations: an overview," J. Geophys. Res. 112, D24S47 (2007).

26. T. J. Schmit et al., "Introducing the next-generation advanced baseline imager on GOES-R," Bull. Am. Meteorol. Soc. 86, 1079-1096 (2005).

Hai Zhang is a research scientist in I.M. Systems Group. He earned his $\mathrm{PhD}$ in atmospheric physics from the University of Maryland Baltimore County. He currently works on aerosol optical depth retrieval and aerosol detection algorithms from satellite sensors. He also develops near-real-time applications of the satellite remote sensed aerosol data for air quality monitoring and forecasting.

Pubu Ciren received his BS and MS degrees in applied meteorology from Nanjing Institute of Meteorology, China and $\mathrm{PhD}$ in atmospheric science from the University of Bergen, Norway. Currently, he is a research scientist with I.M. System Group Inc. in support of satellite aerosol detection algorithm development and validation for JPSS and GOES-R program in NOAA/ NESDIS. His current principal areas of interest include satellite-based smoke/dust detection and its application; and application of satellite remote sensing of aerosol in air quality monitoring.

Shobha Kondragunta is a research scientist at NOAA NESDIS. She develops algorithms to derive air quality products from NOAA's polar orbiting and geostationary satellites. She works with users in the use of satellite products such as aerosol imagery and aerosol/trace gas emissions from fires in operational monitoring and forecasting of air quality.

Istvan Laszlo: Biography is not available. 